\title{
THE CLOSED IDEALS IN A FUNCTION ALGEBRA
}

\author{
BY \\ CHARLES M. STANTON $\left.{ }^{1}\right),\left({ }^{2}\right)$
}

\begin{abstract}
We give a new method of determining the closed ideals in the algebra of functions continuous on a finite Riemann surface and analytic in its interior. Our approach is based on Ahlfors' mapping of a finite Riemann surface onto the unit disc.
\end{abstract}

1. Introduction. Let $W$ be a finite Riemann surface with border $\Gamma$. Let $A(W)$ be the uniform algebra of functions continuous on $W \cup \Gamma$ and analytic on $W$. In this paper we give a new method for determining the closed ideals of $A(W)$, and we show that every closed ideal is the closure of a principal ideal. Our results extend those of Beurling (unpublished) and Rudin [8] for the unit disc. An equivalent description of the closed ideals of $A(W)$ has been obtained by Voichick [11]. Gamelin and Voichick [5] have also proved that every closed ideal is the closure of a principal ideal.

Voichick uses the uniformization theorem to lift $A(W)$ to an algebra of functions on the unit disc. He must then deal with the rather complicated boundary behavior of the uniformizing map. We proceed in the opposite direction, making use of a theorem of Ahlfors [1] to represent $W \cup \Gamma$ as an $n$-sheeted branched covering surface of the closed unit disc. All branching occurs on $W$, thus the Ahlfors covering mapping is a local homeomorphism in a neighborhood of $\Gamma$. It is thus possible to use Ahlfors' theorem to localize the ideal theory of $A(W)$. That is, one need study only those ideals on whose hulls the Ahlfors mapping is a homeomorphism. Since $W$ is an $n$-sheeted covering of the disc one can use classical techniques to show that $A(W)$ is an algebraic extension of the corresponding algebra in the unit disc. We are thus able to apply the ideal theory of the disc algebra to the localized ideal theory of $A(W)$. Alling [3], [4] and Stout [9] - further references can be found in these papers-have made similar use of Ahlfors' theorem to study function theory on finite Riemann surfaces. (See also Alling's review of Stout's paper in Math. Reviews 32 (1966), p. 234, review \#1358.

Received by the editors February 26, 1970.

AMS 1969 subject classifications. Primary 3087, 4655; Secondary 3045.

Key words and phrases. Function algebra, closed ideals, factorization of analytic functions, Riemann surface, Ahlfors mapping.

( ${ }^{1}$ This paper is based on the author's doctoral dissertation written at Stanford University, April 1969, under the direction of Professor H. L. Royden. He thanks Professor Royden for his advice and encouragement.

$\left({ }^{2}\right)$ Research partially supported by Army Research Office, Durham, North Carolina contract DA 31-124-ARO-D-170. 
Aside from the existence of the Ahlfors mapping, the function theoretic arguments thus far are either local, or occur in the disc. To describe the ideal theory of $A(W)$ without mention of an Ahlfors mapping, and to prove that each closed ideal is the closure of a principal ideal, we find it necessary to do some function theory on the Riemann surface. Our development is analogous to Nevanlinna's factorization of functions in the disc.

2. Some function theory. In this section we discuss some of the necessary function theory, beginning with our generalization of Nevanlinna's factorization. We can then state our description of the ideal theory of $A(W)$. A brief discussion of the Ahlfors mapping ends the section. A reference for Riemann surfaces is the book of Ahlfors and Sario [2].

Owing to the difficulty of defining single-valued analytic functions on $W$, we find it more convenient to work with certain harmonic functions in our factorization theory. Let $G(p, q)$ denote the Green's function of $W$ with pole at $q$. We define a harmonic Blaschke function to be a function of the form

$$
-\sum_{k=1}^{\infty} n_{k} G\left(p, q_{k}\right)
$$

where the $n_{k}$ are positive integers and the series converges uniformly on compact subsets of $W \sim\left\{q_{k}\right\}$. If $\omega$ is an open interval on $\Gamma$ containing no limit points of the set $\left\{q_{k}\right\}$, then we can extend $b$ to a harmonic function on $(W \cup \omega) \sim\left\{q_{k}\right\}$ by setting $b(p)=0$ on $\omega$. More generally, the nontangential boundary values of $b$ are zero almost everywhere-with respect to harmonic measure-on $\Gamma$. Observe that in the disc a harmonic Blaschke function is the logarithm of the modulus of a Blaschke product.

Let $H$ be the complex vector space spanned by the positive harmonic functions on $W$. Each function $h$ in $H$ has a unique representation in the form $h=q+s$ where $q$ is a quasi-bounded harmonic function and $s$ is a singular harmonic function. Now $h$ has nontangential boundary values almost everywhere on $\Gamma$, and we also denote by $h$ the function these limits define on $\Gamma$. Then

$$
q(p)=\frac{i}{\pi} \int_{\Gamma} h(q) \partial_{q} G(p, q)
$$

where $\partial=d+i * d-d$ denotes exterior differentiation-and the subscript $q$ indicates differentiation with respect to the second variable. The singular function $s$ has nontangential limits zero almost everywhere on $\Gamma$ with respect to harmonic measure.

2.1. THEOREM. Let $f$ be a bounded analytic function on $W$. Then there exists a harmonic Blaschke function $b$, a singular harmonic function $s$ and a quasi-bounded harmonic function $u$ such that $\log |f|=b+s+u$. The functions $b, s, u$ are uniquely determined. 
Proof. For each $p$ in $W$ let $n_{p}$ denote the order of $f$ at $p$. Set

$$
b(p)=-\sum_{q \in W} G(p, q) .
$$

The function $\log |f|-b$ is in $H$ and has boundary values $\log |f|$. Let $s$ and $u$ be its singular and quasi-bounded components. The uniqueness assertion is evident.

This theorem generalizes Nevanlinna's factorization. For let $f$ be a bounded analytic function in the unit disc and write $\log |f|=b+s+u$ as in the theorem. Take harmonic conjugates of each of $b, s, u$ and exponentiate the resulting analytic functions. There then arises from $b$ a Blaschke product $B$, from $s$ a singular analytic function $S$, and from $u$ an outer function $F$ such that $|f|=|B||S||F|$. Hence $f=B S F$ up to constant factors of modulus one.

Observe that

$$
u(p)=\frac{i}{\pi} \int_{\Gamma} \log |f(q)| \partial_{q} G(p, q)
$$

and that if $f$ is in $A(W)$ then $e^{u}=|f|$ everywhere on $\Gamma$. Also $\log |f(p)| \leqq u(p)$ on $W$, and so $s(p) \leqq 0$ on $W$. In particular if $\omega$ is an open interval on $\Gamma$ such that $f$ is continuous and does not vanish on $\omega$, then we can extend both $b$ and $s$ to be harmonic on $\omega$ by setting them equal to zero there. We call $s$ the singular harmonic part of $f$.

We can prescribe the Blaschke function and the singular harmonic part of a bounded analytic function on $W$. For this purpose a theorem of Wermer [10] is convenient.

2.2. THEOREM. There are functions $Z_{1}, \ldots, Z_{N}$, analytic and nowhere vanishing on $W \cup \Gamma$, such that if $U$ is a harmonic function on $W$, there exist real constants $\alpha_{1}, \ldots, \alpha_{N}$ such that the function $U^{\prime}=U-\sum_{k=1}^{N} \alpha_{k} \log \left|Z_{k}\right|$ has a single-valued conjugate $V^{\prime}$ on $W$. If $U$ is of class $C^{2}$ on an open interval on $\Gamma$ then $V^{\prime}$ is continuous there. If $U$ is harmonic on such an interval, so is $V^{\prime}$.

Applying Wermer's theorem to a Blaschke function $b$ we obtain a multiplevalued analytic function whose only periods are of the form $2 n \pi i$ around the poles of $b$. We exponentiate this function and get a bounded analytic function $B$ with zeros at the poles of $b$. The singular harmonic part of $B$ is zero, for $\log |B|$ $=b-\sum \alpha_{k} \log \left|Z_{k}\right|$ and each function $\log \left|Z_{k}\right|$ is quasi-bounded. Let $s$ be a nonpositive singular harmonic function and let $\beta_{1}, \ldots, \beta_{N}$ be real constants such that $s^{\prime}=s-\sum_{k=1}^{N} \beta_{k} \log \left|Z_{k}\right|$ has a single-valued harmonic conjugate $t^{\prime}$. Let $S$ $=\exp \left(s^{\prime}+i t^{\prime}\right)$. Then $S$ is a nowhere vanishing bounded analytic function on $W$ whose singular harmonic part is $s$. Moreover $S$ is analytic everywhere on $\Gamma$ that $s$ is harmonic. The function $B S$ is thus a bounded analytic function on $W$ whose Blaschke part is $b$ and whose singular harmonic part is $s$. 
Let $\mathscr{I}$ be a closed ideal in $A(W)$ and let $E$ be its hull; that is $E=\{p \in W \cup \Gamma: f(p)$ $=0$ for every $f \in \mathscr{I}\}$. We denote the order of $f$ at a point $p$ of $W$ by $\operatorname{ord}_{p} f$, and we define the order of $\mathscr{I}$ at $p$ to be

$$
\operatorname{ord}_{p} \mathscr{I}=\min \left\{\operatorname{ord}_{p} f: f \in \mathscr{I}\right\} \text {. }
$$

For each $f$ in $\mathscr{I}$ let $s_{f}$ be the singular harmonic part of $f$. The singular harmonic part $s$ of $\mathscr{I}$ is the least harmonic majorant of the family $\left\{s_{f}: f \in \mathscr{I}\right\}$. If $\omega$ is an open interval on $\Gamma$ such that there is a function $f$ in $\mathscr{I}$ which does not vanish on $\omega$, then we can extend $s$ harmonically to $\omega$ by setting $s=0$ there. For $s_{f} \leqq s \leqq 0$ and $s_{f}=0$ on $\omega$.

We can now state one description of the closed ideals in $A(W)$.

Theorem I. Let $\mathscr{I}$ be a closed ideal in $A(W)$, let $E$ be the hull of $\mathscr{I}$ and let $s$ be the singular harmonic part of $\mathscr{I}$. Let $f$ be in $A(W)$ and let $s_{f}$ be the singular harmonic part of $f$. Then $f$ is in $\mathscr{I}$ if and only if

(i) $f$ vanishes on $E$,

(ii) $\operatorname{ord}_{p} f \geqq \operatorname{ord}_{p} \mathscr{I}$ for all $p$ in $W$,

(iii) $s_{f} \leqq s$.

Corollary (Gamelin and Voichick [5]). Let $\mathscr{I}$ be a closed ideal in $A(W)$. Then $\mathscr{I}$ is the closure of a principal ideal.

Proof. Let $s$ be the singular harmonic part of $\mathscr{I}$ and let $b$ be the Blaschke function

$$
b(p)=-\sum_{q \in W} \operatorname{ord}_{q} \mathscr{I} \cdot G(p, q)
$$

According to the remarks following Wermer's theorem there is a bounded analytic function $\Phi$ on $W$ such that the singular harmonic part and Blaschke function of $\Phi$ are $s$ and $b$. Moreover $\Phi$ is analytic on $W \cup(\Gamma \sim E)$. There is an analytic function $f$ in $A(W)$ such that $f$ vanishes only on $\Gamma \cap E$ and $\log |f|$ is quasi-bounded. (The proof of this generalization of Fatou's theorem is analogous to the proof in the case of the disc.) The closed ideal generated by $f \Phi$ is $\mathscr{I}$.

Theorem $I$ is a generalization of the theorem of Beurling and Rudin for the disc. Let $U$ denote the open unit disc, $T$ the unit circle and $D=U \cup T$. Let $A(U)$ denote the disc algebra. If $\mathscr{I}$ is a closed ideal in $A(U)$, we form a Blaschke product $B$ from its interior zeros, and a singular analytic function $S$ from its singular harmonic part. For a function $f$ in $A(U)$ conditions (ii) and (iii) of Theorem I are equivalent to the condition that $f / B S$ be bounded. Hence in the case of the disc Theorem I reduces to the theorem of Beurling and Rudin.

TheOREM (Beurling AND Rudin). Let $I$ be a closed ideal in $A(U)$ and let $E$ be the hull of $I$. There is an inner function $\Phi$ such that a function $f$ in $A(U)$ is in I if and only if $f$ vanishes on $E$ and $f \Phi^{-1}$ is bounded. 
We shall prove Theorem I by describing the ideal theory of $A(W)$ in terms of the ideal theory of the disc algebra. Our argument rests on the following theorem of Ahlfors [1].

THEOREM. There is a function $f$ which is analytic on $W \cup \Gamma$ and which maps $W$ onto $U$ and $\Gamma$ onto $T$.

This theorem is also proved in Royden [7]. A function $f$ of the kind described in this theorem is called an Ahlfors mapping. An Ahlfors mapping has no branch points in $\Gamma$, thus its branch points form a finite subset of $W$, and it is a local homeomorphism in a neighborhood of $\Gamma$. An Ahlfors mapping also takes every value the same number of times.

3. Localization of the ideal theory of $A(W)$. For the remainder of the paper we let $z$ denote a fixed Ahlfors mapping of $W \cup \Gamma$ onto $D$. Observe that $z$ embeds $A(U)$ in $A(W)$ as a closed subalgebra. A function $a$ in $A(U)$ is identified with the function $a \circ z$ in $A(W)$. Let $n$ be the multiplicity of $z$, and let $B$ be the image under $z$ of the set of branch points of $z$. Now $z$ realizes $W \cup \Gamma$ as an $n$-sheeted branched covering surface of $D$. Away from $z^{-1}[B]$ this covering is even in the sense that each point of $D \sim B$ has a neighborhood $V$ such that $z^{-1}[V]$ consists of $n$ components, each of which is mapped homeomorphically onto $V$. We call such a neighborhood of $V$ evenly covered; note that any connected subneighborhood of an evenly covered neighborhood is evenly covered.

Let $\mathscr{I}$ be a closed ideal in $A(W)$ and let $E$ be its hull. Our plan in this section and the next is to divide $E$ into a finite disjoint union of closed sets such that $z$ is one-to-one on all but perhaps one-that one being a finite subset of $W$. We then apply the Silov idempotent theorem to show that $\mathscr{I}$ is the intersection of uniquely determined closed ideals with these sets as hulls. In the next section we show that these ideals can be described in terms of the ideal theory of $A(U)$. We are thus able to reduce Theorem I to the ideal theory of $A(U)$.

We call a closed subset $E$ of $W \cup \Gamma$ simple with respect to $z$ if $z$ is one-to-one on $E$ and $z[E] \cap B=\varnothing$. Let $E$ be a closed subset of $W \cup \Gamma$. We say that $E$ has a simple decomposition with respect to $z$ if there are pairwise disjoint closed subsets $E_{0}, E_{1}, \ldots, E_{m}$ of $E$ such that $E=E_{0} \cup E_{1} \cup \cdots \cup E_{m}, E_{0}=E \cap z^{-1}[B]$ and each of $E_{1}, \ldots, E_{m}$ is simple with respect to $z$. We say that a closed ideal in $A(W)$ is simple with respect to $z$ if its hull is simple. Let $\mathscr{I}$ be a closed ideal in $A(W)$ with hull $E$. We say that the closed ideals $\mathscr{I}_{0}, \mathscr{I}_{1}, \ldots, \mathscr{I}_{m}$ form a simple decomposition of $\mathscr{I}$ with respect to $z$ if $\mathscr{I}=\mathscr{I}_{0} \cap \mathscr{I}_{1} \cap \cdots \cap \mathscr{I}_{m}$ and the hulls of the $\mathscr{I}_{k}$ form a simple decomposition of $E$, that is, the hull of $\mathscr{I}_{0}$ is $z^{-1}[B] \cap E$ and $\mathscr{I}_{1}, \ldots, \mathscr{I}_{m}$ are simple ideals with pairwise disjoint hulls. We mention explicitly that we allow some of the sets $E_{k}$ to be empty; if $E_{k}=\varnothing$ then $\mathscr{I}_{k}=A(W)$.

3.1. Lemma. Let $\mathscr{I}$ be a closed ideal in $A(W)$, and let $E$ be the hull of $\mathscr{I}$. Let $z$ be an Ahlfors mapping of $W$. Then there is a simple decomposition of $E$ with respect to $z$. 
Proof. Let $F=z[E]$. Then $F$ is a closed subset of $D, F \cap U$ is discrete and $F \cap T$ is nowhere dense in $T$. Each point $\zeta$ in $F \cap T$ lies in an open interval $I$ on $T$ such that the endpoints of $I$ do not belong to $F$ and $I$ is contained in an evenly covered neighborhood. Finitely many such intervals $I_{1}, \ldots, I_{p}$ cover $F \cap T$ and these intervals may be chosen to be disjoint.

Choose $r$ so that $B$ lies in $\{\zeta \in D:|\zeta|<r\}, F$ does not meet $\{\zeta \in D:|\zeta|=r\}$ and each of the neighborhoods

$$
V_{k}=\left\{\rho e^{i \theta} \in D: r<\rho<1, e^{i \theta} \in I_{k}\right\}
$$

$k=1, \ldots, p$, is evenly covered by $z$. For each $k=1, \ldots, p$, let $E_{j, k}, j=1, \ldots, n$, be the components of $z^{-1}\left[V_{k}\right]$. Let $E_{0}=E \cap z^{-1}[B]$ and $E_{1}=E \sim\left(E_{0} \cup \cup E_{j k}\right)$. Now $E_{1}$ is a finite set, and so $E_{0}$, the sets $\{p\}$ where $p$ is in $E_{1}$, and the sets $E_{j k} \cap E$ form a simple decomposition of $E$.

Corresponding to each simple decomposition of the hull there is a uniquely determined simple decomposition of the ideal. This argument depends on the Silov idempotent theorem, and we prefer to present it in the more general context of Banach algebras.

3.2. Lemma. Let $A$ be a commutative Banach algebra with identity e, and let $S$ be maximal ideal space of $A$. Let $S_{1}, \ldots, S_{m}$ be pairwise disjoint closed nonvoid subsets of $S$ such that $S=S_{1} \cup \ldots \cup S_{m}$. Then there exist closed ideals $I_{1}, \ldots, I_{m}$ in $A$ such that

(i) $S_{k}$ is the hull of $I_{k}, k=1, \ldots, m$,

(ii) $\cap I_{k}=0$.

Furthermore, if $J_{1}, \ldots, J_{m}$ are closed ideals in A satisfying (i) and (ii), then $I_{k}=J_{k}$.

Proof. Let $e_{1}, \ldots, e_{m}$ be idempotents in $A$ such that $e_{1}+\cdots+e_{m}=e$ and the Gelfand transform of $e_{k}$ is the characteristic function of $S_{k}$. Let $I_{k}=\left\{f \in A: f e_{k}=0\right\}$. Then $I_{k}$ is a closed ideal in $A$ with hull $S_{k}$ and $\bigcap I_{k}=0$.

For the uniqueness we may assume that $J_{k} \subset I_{k}$ for each $k$ because we can replace each $J_{k}$ by $I_{k} \cap J_{k}$. Let $I^{\prime}$ and $J^{\prime}$ be the smallest closed ideals in $A$ which contain $I_{2} \cup \ldots \cup I_{m}$ and $J_{2} \cup \ldots \cup J_{m}$ respectively, Then $I^{\prime}$ and $J^{\prime}$ have hulls $S \sim S_{1}$. Since $I_{1}$ and $I^{\prime}$ have disjoint hulls and $I_{1} \cap I^{\prime}=0, A=I_{1} \oplus I^{\prime}$. Similarly $A=J_{1} \oplus J^{\prime}$. Since $J_{1} \subset I_{1}$ and $J^{\prime} \subset I^{\prime}$ it follows that $I_{1}=J_{1}$. Hence $I_{k}=J_{k}, k=1, \ldots, m$, by the same argument.

3.3. Proposition. Let $z$ be an Ahlfors mapping of $W$. Let $\mathscr{I}$ be a closed ideal in $A(W)$, and let $E=E_{0} \cup E_{1} \cup \ldots \cup E_{m}$ be a simple decomposition of its hull with respect to $z$. Then there is exactly one simple decomposition $\mathscr{I}=\mathscr{I}_{0} \cap \mathscr{I}_{1} \cap \ldots$ $\cap \mathscr{I}_{m}$ of $\mathscr{I}$ with respect to $z$ such that the hull of $\mathscr{I}_{k}$ is $E_{k}, k=1, \ldots, m$.

We obtain Proposition 3.3 by applying Lemma 3.2 to the algebra $A(W) / \mathscr{I}$, whose maximal ideal space is $E$, and then using the natural one-to-one correspondence between closed ideals in $A(W) / \mathscr{I}$ and closed ideals in $A(W)$ which contain $\mathscr{I}$. 
The fact that the ideals in a simple decomposition are uniquely determined by their hulls leads to the following remark. Let $F$ be a set occurring in a simple decomposition of the hull of a closed ideal $\mathscr{I}$, and let $\mathscr{I}_{F}$ be the corresponding ideal. Then $\mathscr{I}_{F}$ is the smallest closed ideal in $A(W)$ whose hull is $F$ and which contains $\mathscr{I}$.

4. Proof of Theorem I. The Ahlfors mapping and the Silov idempotent theorem allow us to apply the ideal theory of $A(U)$ to describe the simple ideals in $A(W)$. By borrowing techniques from the theory of closed Riemann surfaces, we can show that $A(W)$ is an algebraic extension of $A(U)$. Indeed for $f$ in $A(W)$ there exist $a_{1}, \ldots, a_{n}$ in $A(U)$ such that

$$
f^{n}+a_{1} f^{n-1}+\cdots+a_{n}=0
$$

and for $\zeta$ in $D \sim B,(-1)^{k} a_{k}(\zeta)$ is the $k$ th elementary symmetric function of the values of $f$ on $z^{-1}[\zeta]$ (cf. Alling [3]). Equation (1) is determined by its construction and we shall call it the standard equation for $f$ with respect to $z$.

Let $\mathscr{I}$ be a closed ideal in $A(W)$ with hull $E$. Let $I=\mathscr{I} \cap A(U)$. Since $A(U)$ is a closed subalgebra of $A(W), I$ is a closed ideal in $A(U)$. Moreover the hull of $I$ is $z[E]$. For let $F$ be the hull of $I$. Then $z[E] \subset F$. To obtain the reverse inclusion we let $\zeta$ be in $D \sim z[E]$. Then there is an $f$ in $\mathscr{I}$ such that $f$ vanishes at no point of $z^{-1}[\zeta]$. Let $f^{n}+a_{1} f^{n-1}+\cdots+a_{n}=0$ be the standard equation for $f$. Then $a_{n}$ is in $I$ and $a_{n}(\zeta) \neq 0$. Thus $\zeta$ is not in $F$, and so $F=z[E]$. A refinement of this argument shows that if $\zeta$ is in $F \sim B$ and $z^{-1}[\zeta] \cap E$ consists of exactly one point $p$, then $\operatorname{ord}_{p} \mathscr{I}=\operatorname{ord}_{\zeta} I$. We need only choose $f$ not to vanish on $z^{-1}[\zeta] \sim\{p\}$ and so that $\operatorname{ord}_{p} f=\operatorname{ord}_{p} \mathscr{I}$. Then $\operatorname{ord}_{\zeta} a_{n}=\operatorname{ord}_{p} f=\operatorname{ord}_{p} \mathscr{I}$.

Let $\mathscr{I}$ be a closed ideal in $A(W)$ with hull $E$, and let $I=\mathscr{I} \cap A(U)$. Then $I$ is a closed ideal in $A(U)$ with hull $z[E]$. On the other hand let $J$ be a closed ideal in $A(U)$ with hull $F$ and inner function $\Phi$. Let $\mathscr{J}$ be the smallest closed ideal in $A(W)$ which contains $J$. Then the hull of $\mathscr{J}$ is $z^{-1}[F]$. Moreover if $f$ is in $\mathscr{J}$, then $f \Phi^{-1}$ is a bounded analytic function on $W$. For $\mathscr{J}$ is the closure of the set of all functions $f$ of the form $f=a_{1} f_{1}+\cdots+a_{m} f_{m}$ where $a_{k} \in J$ and $f_{k} \in A(W)$. The assertion follows because $f \Phi^{-1}$ is bounded for each such function $f$ and this property is preserved under uniform convergence.

The next lemma is the main step in our treatment of simple ideals.

4.1. Lemma. Let $z$ be an Ahlfors mapping of $W$ and let $\mathscr{I}$ be a closed ideal in $A(W)$ which is simple with respect to $z$. For each $f$ in $A(W)$ there is an a in $A(U)$ such that $f-a \circ z$ is in $\mathscr{I}$.

The following lemma is useful in the proof of Lemma 4.1. It is nothing but the Silov idempotent theorem stated in an immediately applicable form.

4.2. LemMA. Let $z$ be an Ahlfors mapping of $W$ and let $A(U)$ be embedded in $A(W)$ by means of $z$. Let $I$ be a closed ideal in $A(U)$ with hull $F$ and inner function $\Phi$. Let $E$ be a closed subset of $z^{-1}[F]$ such that $z$ is one-to-one on $E$ and $z[E]=F$. Let $\mathscr{J}$ 
be the smallest closed ideal in $A(W)$ which contains $I$. Then there is a function $h$ in $A(W)$ such that

(i) $h(p)=1$ for $p$ in $E$ and $h(p)=0$ for $p$ in $z^{-1}[F] \sim E$.

(ii) $h(1-h)$ is in $\mathscr{J}$.

(iii) $h(1-h) \Phi^{-1}$ is a bounded analytic function on $W$.

Proof. The hull of $\mathscr{J}$ is $z^{-1}[F]$, so the maximal ideal space of $A(W) / \mathscr{J}$ is $z^{-1}[F]$. Now $z^{-1}[F] \sim E$ is a closed subset of $W \cup \Gamma$. (This fact follows from the covering properties of the mapping $z$. Its proof is similar to the proof of Lemma 3.2.) Thus $E$ and $z^{-1}[F] \sim E$ disconnect the maximal ideal space of $A(W) / \mathscr{J}$. Hence $A(W) / \mathscr{J}$ has idempotents $e$ and $1-e$ whose Gelfand transforms are the characteristic functions of $E$ and $z^{-1}[F] \sim E$. Let $h$ belong to the coset $e$ in $A(W)$. Then $h=1$ on $E$ and $h=0$ on $z^{-1}[F] \sim E$. Since $e(1-e)=0$ in $A(W) / \mathscr{J}, h(1-h)$ is in $\mathscr{J}$. By our earlier remark, $h(1-h) \Phi^{-1}$ is bounded.

Proof of Lemma 4.1. Embed $A(U)$ in $A(W)$ by means of $z$. Let $E$ be the hull of $\mathscr{I}$ and let $I=\mathscr{I} \cap A(U)$. Let $\Phi$ be the inner function of $I$ and let $\mathscr{J}$ be the smallest closed ideal in $A(W)$ which contains $I$. Let $h$ be a function as in Lemma 4.2. Since $h$ does not vanish on the hull of $\mathscr{I}, h$ is an invertible element in the algebra $A(W) / \mathscr{I}$. In this algebra $h(1-h)=0$ for $h(1-h)$ is in $\mathscr{J}$ and $\mathscr{J} \subset \mathscr{I}$. Thus $1-h=0$ in $A(W) / \mathscr{I}$; that is, $1-h$ is in $\mathscr{I}$.

We assume first that $f$ does not vanish on $E$, and we prove that there is a function $a$ in $A(U)$ such that $f h-a$ is in $\mathscr{I}$. Let the standard equation for $f h$ be

$$
(f h)^{n}+q_{1}(f h)^{n-1}+q_{2}(f h)^{n-2}+\cdots+q_{n}=0 .
$$

The coefficients $q_{2}, \ldots, q_{n}$ belong to $I$, for there is a relatively open set $V$ in $D$ and there are relatively open sets $V_{1}, \ldots, V_{n}$ in $W \cup \Gamma$ such that $z$ maps each $V_{k}$ homeomorphically onto $V, E \subset V_{1}$ and $z^{-1}[z[E]] \subset V_{2} \cup \cdots \cup V_{n}$. We may assume that $h$ does not vanish on $V_{1}$ and $1-h$ does not vanish on $V_{2} \cup \cdots \cup V_{n}$. Consequently $h \Phi^{-1}$ is bounded on $\left(V_{2} \cup \cdots \cup V_{n}\right) \cap W$.

For $\zeta$ in $V$ let $p_{k}$ be the point of $V_{k}$ which lies over $\zeta$. The coefficients $q_{2}, \ldots, q_{n}$ are given by the formulas

$$
\begin{gathered}
q_{2}(\zeta)=\sum_{i \neq j} f\left(p_{i}\right) h\left(p_{i}\right) f\left(p_{j}\right) h\left(p_{j}\right) \\
\vdots \\
q_{n}(\zeta)=(-1)^{n} \prod_{j} f\left(p_{j}\right) h\left(p_{j}\right) .
\end{gathered}
$$

Thus for each $k=2, \ldots, n, q \Phi^{-1}$ is bounded on $V \cap U$-and so on $U$-and $q_{k}$ vanishes on $z[E]$. Therefore $q_{2}, \ldots, q_{n}$ are in $I$. It now follows that $(f h)^{n}+q_{1}(f h)^{n-1}$ is in $\mathscr{I}$. Since $f h$ does not vanish on $E,(f h)^{n-1}$ is invertible modulo $\mathscr{I}$ and so $f h+q_{1}$ is in $\mathscr{I}$.

We have shown that if $f$ does not vanish on $E$, then there is an $a$ in $A(U)$ such that $f h-a$ is in $\mathscr{I}$. If $f$ is any function in $A(W)$ there is a constant $\alpha$ such that $f+\alpha$ 
does not vanish on $E$. There is an $a$ in $A(U)$ such that $(f+\alpha) h-a$ is in $\mathscr{I}$. Now $(f+\alpha)(1-h)$ is in $\mathscr{I}$, thus $f+\alpha-a$ is in $\mathscr{I}$. Since $\alpha-a$ is in $A(U)$ the lemma is proved.

4.3. Proposition. Let $z$ be an Ahlfors mapping of $W$ and let $A(U)$ be embedded in $A(W)$ by means of $z$. Let $\mathscr{I}$ and $\mathscr{J}$ be closed ideals in $A(W)$ which are both simple with respect to $z$. Assume that $\mathscr{I}$ and $\mathscr{J}$ have the same hull, and that $\mathscr{I} \cap A(U)$ $=\mathscr{J} \cap A(U)$. Then $\mathscr{I}=\mathscr{J}$.

Proof. We may assume that $\mathscr{I} \subset \mathscr{J}$ because we can replace $\mathscr{I}$ by $\mathscr{I} \cap \mathscr{I}$. We shall show that $\mathscr{J} \subset \mathscr{I}$. Let $f$ be in $\mathscr{J}$. There is an $a$ in $A(U)$ such that $f-a$ is in $\mathscr{I}$ by Lemma 4.1. Then $a$ is in $\mathscr{J} \cap A(U)$, so $a$ is in $\mathscr{I}$. Thus $f$ is in $\mathscr{I}$, and so $\mathscr{I}=\mathscr{J}$.

Before applying Proposition 4.3 to Theorem I, we shall need the following lemma.

4.4. Lemma. Let $E$ be a closed subset of $\Gamma$ of harmonic measure zero and let $s$ be a nonpositive singular harmonic function on $W$. Assume that $s$ extends harmonically to $(W \cup \Gamma) \sim E$. For each $f$ in $A(W)$ let $s_{f}$ be the singular harmonic part of $f$. The set $\mathscr{I}$ of all $f$ in $A(W)$ such that $s_{f} \leqq s$ is a closed ideal in $A(W)$.

Proof. In the notation of Wermer's theorem there are real constants $\alpha_{1}, \ldots, \alpha_{N}$ such that $s^{\prime}=s-\sum \alpha_{k} \log \left|Z_{k}\right|$ has a single valued harmonic conjugate $t^{\prime}$ and $s^{\prime}+t^{\prime}$ is analytic on $(W \cup \Gamma) \sim E$. Let $S=\exp \left(s^{\prime}+i t^{\prime}\right)$. Then $S$ is analytic on $(W \cup \Gamma) \sim E$ and $|S|=\left|Z_{1}\right|^{\alpha_{1}} \ldots\left|Z_{n}\right|^{\alpha_{N}}$ almost everywhere on $\Gamma$.

To say that $s_{f} \leqq s$ is equivalent to saying that $f S^{-1}$ is bounded. This last property is preserved under sums, products and uniform convergence. Thus the lemma is proved.

Proof of Theorem I. We first prove Theorem I under the additional assumption that the hull of $\mathscr{I}$ is a finite subset of $W$. Let $\psi$ be a function in $A(W)$ such that $\psi$ is analytic on $W \cup \Gamma, \operatorname{ord}_{p} \psi=\operatorname{ord}_{p} \mathscr{I}$ for each $p$ in $W$ and $\psi$ does not vanish on $\Gamma$. Let $\mathscr{J}=\left\{f \in A(W): f=g \psi^{-1}\right.$ for some $\left.g \in \mathscr{I}\right\}$. Then $\mathscr{J}$ is an ideal in $A(W)$ with empty hull. Let $J=\mathscr{J} \cap A(U)$. Then $J$ is an ideal in $A(U)$ with empty hull, and so $J=A(U)$. Thus $\mathscr{J}=A(W)$. Therefore $\mathscr{I}$ is the set of all functions $f$ in $A(W)$ such that $\operatorname{ord}_{p} f \geqq \operatorname{ord}_{p} \mathscr{I}$ for each $p$ in $W$. Since the singular harmonic part of $\mathscr{I}$ is zero, Theorem $\mathrm{I}$ is proved under the additional assumption that the hull of $\mathscr{I}$ is a finite subset of $W$.

We next assume that $\mathscr{I}$ is a simple ideal in $A(W)$. Let $I=\mathscr{I} \cap A(U)$, and let $s_{1}$ be the least harmonic majorant of $\left\{s_{f}: f \in I\right\}$. Then $s_{1} \leqq s$. Let $\mathscr{J}$ be the set of functions $f$ in $A(W)$ which satisfy conditions (i), (ii), (iii) of Theorem I and the condition $s_{f} \leqq s_{1}$. Then $\mathscr{J}$ is a closed ideal in $A(W)$ with hull $E$ and $I=\mathscr{J} \cap A(U)$. Thus $\mathscr{I}=\mathscr{J}$ by Proposition 4.3, and so $s_{1}=s$. Thus Theorem I is proved under the additional hypothesis that $\mathscr{I}$ is simple.

In the general case we let $E=E_{0} \cup E_{1} \cup \cdots \cup E_{m}$ and $\mathscr{I}=\mathscr{I}_{0} \cap \mathscr{I}_{1} \cap \cdots \cap \mathscr{I}_{m}$ be corresponding simple decompositions of $E$ and $\mathscr{I}$, and we let $s_{1}, \ldots, s_{m}$ be the 
singular harmonic parts of $\mathscr{I}_{1}, \ldots, \mathscr{I}_{m}$. If $f$ is in $\mathscr{I}$ then $f$ is in each $\mathscr{I}_{k}$ and so $s_{f} \leqq s_{k}$. The sets $E_{k}$ are disjoint and each $s_{k}$ is a nonpositive singular harmonic function which is harmonic on $W \cup\left(\Gamma \sim E_{k}\right)$. Thus the greatest harmonic minorant of $\left\{s_{1}, \ldots, s_{m}\right\}$ is $s_{1}+\cdots+s_{m}$. Hence $s_{f} \leqq s_{1}+\cdots+s_{m}$ for each $f$ in $\mathscr{I}$, and $s \leqq s_{1}+\cdots+s_{m}$.

Let $\mathscr{J}$ be the collection of all $f$ in $A(W)$ which satisfy (i), (ii), (iii). Then $\mathscr{J}$ is a closed ideal in $A(W)$ and $\mathscr{I} \subset \mathscr{J}$. If $f$ is in $\mathscr{J}$ then $f$ satisfies (i), (ii), and $s_{f} \leqq s \leqq s_{k}$ for each $k$. Hence $f$ is in each $\mathscr{I}_{k}$ and so $f$ is in $\mathscr{I}$.

5. Ideal theory and the Ahlfors mapping. It is of some interest to describe the ideal theory of $A(W)$ in terms of the Ahlfors mapping as this approach involves a minimum of global function theory on $W$. In this section we sketch briefly how this can be done. Let $\mathscr{I}$ be a closed ideal in $A(W)$ and let $E$ be the hull of $\mathscr{I}$. Our first task is to construct a positive Baire measure $\mu$ on $\Gamma$ corresponding to $\mathscr{I}$. We first assume that $\mathscr{I}$ is simple with respect to $z$. Let $I=\mathscr{I} \cap A(U) ; I$ is a closed ideal in $A(U)$. Let $\nu$ be the positive Baire measure associated with the inner function of $I$. Since $z$ maps $E \cap \Gamma$ homeomorphically onto $z[E] \cap T$ and since $v$ is supported by $z[E] \cap T$, it follows that there is a positive Baire measure $\mu$ on $\Gamma$ with support in $E \cap \Gamma$ defined by $\mu(S)=\nu[z(S)]$ for every Baire set $S$ in $E \cap \Gamma$.

In the general case let $E=E_{0} \cup E_{1} \cup \cdots \cup E_{m}$ be a simple decomposition of $E$ with respect to $z$, and let $\mathscr{I}=\mathscr{I}_{0} \cap \mathscr{I}_{1} \cap \cdots \cap \mathscr{I}_{m}$ be the corresponding simple decomposition of $\mathscr{I}$. Let $\mu_{k}$ be the measure determined by $\mathscr{I}_{k}$ in the above manner, and let $\mu=\mu_{1}+\cdots+\mu_{m}$. Each $\mu_{k}$ has support in $E_{k} \cap \Gamma$, and so $\mu$ is a positive Baire measure on $\Gamma$ with support in $E \cap \Gamma$.

The measure $\mu$ depends only on $\mathscr{I}$ and $z$, and is independent of the particular simple decomposition used to construct it. This can be seen by taking a common refinement of two simple decompositions and applying Proposition 3.3 on the uniqueness of simple decompositions. The argument is straightforward, although somewhat tedious, and we omit it.

Let $\mathscr{I}$ be a closed ideal in $A(W)$ and let $\mu$ be the measure determined by $\mathscr{I}$ in the manner described above. We call $\mu$ the measure of $\mathscr{I}$ with respect to $z$. If $f$ is in $A(W)$ we define the measure of $f$ with respect to $z$ to be the measure of the closure of the principal ideal generated by $f$. Let $\lambda$ be a positive Baire measure on $\Gamma$ and let $\nu$ be the measure of $f$ with respect to $z$. We say that $f$ is a multiple of $\lambda$ with respect to $z$ if $\nu \geqq \lambda$.

We can now describe the closed ideals of $A(W)$ in terms of $z$.

THEOREM II. Let $\mathscr{I}$ be a closed ideal in $A(W)$. Let $E$ be the hull of $\mathscr{I}$. Let $z$ be an Ahlfors mapping of $A(W)$, and let $\mu$ be the measure of $\mathscr{I}$ with respect to $z$. Then $\mathscr{I}$ is the set of functions $f$ in $A(W)$ such that

(i) $f$ vanishes on $E$,

(ii) $\operatorname{ord}_{p} f \geqq \operatorname{ord}_{p} \mathscr{I}$ for all $p$ in $W$,

(iii) $f$ is a multiple of $\mu$ with respect to $z$. 
Several preliminary results are necessary before we can prove Theorem II.

5.1. Lemma. Let $z$ be an Ahlfors mapping of $W$ and let $A(U)$ be embedded in $A(W)$ by means of $z$. Let $I$ be a closed ideal in $A(U)$ with hull $F$ and assume that $z$ is unbranched on $z^{-1}[F]$. Let $E$ be a subset of $W \cup \Gamma$ which $z$ maps homeomorphically onto $F$. Then there is exactly one closed ideal $\mathscr{I}$ in $A(W)$ such that the hull of $\mathscr{I}$ is $E$ and $\mathscr{I} \cap A(U)=I$.

Proof. Let $\mathscr{J}$ be the smallest closed ideal in $A(W)$ which contains $I$. Let $h$ be as in Lemma 4.2. Let $\mathscr{I}$ be the smallest closed ideal in $A(W)$ which contains $I$ and $1-h$. The hull of $I$ is $E$, thus $\mathscr{I}$ is simple. Each $f$ in $\mathscr{I}$ vanishes on $E$, and $f \Phi^{-1}$ is bounded in a neighborhood of $E$. (Here $\Phi$ is the inner function of $I$.) Therefore if $a$ is in $A(U) \cap \mathscr{I}, a$ vanishes on $F$ and $a \Phi^{-1}$ is bounded on $U$. Thus $I=\mathscr{I} \cap A(U)$. The uniqueness of $\mathscr{I}$ has been proved in Proposition 4.3.

It is now possible to prove that if $\mu$ is a positive Baire measure on $\Gamma$ then the set of functions in $A(W)$ which are multiples of $\mu$ is a closed ideal in $A(W)$. It is clear how this can be done if the support of $\mu$ is simple with respect to $z$. The general case then follows by taking a simple decomposition of the support of $\mu$.

We are now ready to complete the proof of Theorem II. As we have seen in proving Theorem I, Theorem II is valid under the additional assumption that $\mathscr{I}$ is an ideal whose hull is a finite subset of $W$. We next assume that $\mathscr{I}$ is simple with respect to $z$. Let $E$ be the hull of $\mathscr{I}$ and let $\mu$ be the measure of $\mathscr{I}$ with respect to $z$. Let $I=\mathscr{I} \cap A(U)$; the hull of $I$ is $z[E]$. Let $\zeta$ be in $z[E] \cap U$ and let $p$ be the point of $E$ with $z(p)=\zeta$. Then, as we have previously remarked, $\operatorname{ord}_{p} \mathscr{I}=\operatorname{ord}_{p} I$. Let $v$ be the measure on the circle defined by $\nu[S]=\mu\left(z^{-1}[S]\right)$. Then $\nu$ is the measure of the inner function of $I$. Let $\mathscr{J}$ be the set of functions $f$ in $A(W)$ which satisfy conditions (i), (ii), (iii) of Theorem II. Now $\mathscr{J}$ is a closed ideal in $A(W)$. Since $\mathscr{I} \subset \mathscr{J}$, the hull of $\mathscr{J}$ is $E$ and $\mathscr{J}$ is simple. Let $J=\mathscr{J} \cap A(U)$. The data for $I$ and $J$ are the same, thus $I=J$. By Proposition 4.3, $\mathscr{I}=\mathscr{J}$.

Finally, let $\mathscr{I}$ be an arbitrary closed ideal in $A(W)$. Let $E$ be the hull of $\mathscr{I}$, and let $\mu$ be the measure of $\mathscr{I}$. Let $\mathscr{J}$ be the set of all functions satisfying conditions (i), (ii), (iii) of Theorem II. Then $\mathscr{J}$ is a closed ideal and $\mathscr{I} \subset \mathscr{J}$. Let $\mathscr{I}=\mathscr{I}_{0} \cap \mathscr{I}_{1}$ $\cap \cdots \cap \mathscr{I}_{m}$ be a simple decomposition of $\mathscr{I}$. Now $\mathscr{J} \subset \mathscr{I}_{k}, k=0,1, \ldots, m$, because the theorem has been established for the ideals $\mathscr{I}_{k}$. Thus $\mathscr{J} \subset \mathscr{I}$ and $\mathscr{J}=\mathscr{I}$.

Added in proof. Professor Norman Alling has called my attention to the following related paper: B. V. Limaye, Blaschke products for finite Riemann surfaces, Studia Math. 34 (1970).

\section{REFERENCES}

1. L. V. Ahlfors, Open Riemann surfaces and extremal problems on compact subregions, Comment. Math. Helv. 24 (1950), 100-134. MR 12, 90; MR 13, 1138.

2. L. V. Ahlfors and L. Sario, Riemann surfaces, Princeton Math. Series, no. 26, Princeton Univ. Press, Princeton, N. J., 1960. MR 22 \#5729.

3. N. L. Alling, Extensions of meromorphic function rings over non-compact Riemann surfaces. I, Math. Z. 89 (1965), 273-299. MR 32 \#6252. 
4. N. L. Alling, Extensions of meromorphic function rings over non-compact Riemann surfaces. II, Math. Z. 93 (1966), 345-394. MR 33 \#7572.

5. T. W. Gamelin and M. Voichick, Extreme points in spaces of analytic functions, Canad. J. Math. 20 (1968), 919-928. MR 37 \#3023.

6. K. M. Hoffman, Banach spaces of analytic functions, Prentice-Hall Series in Modern Analysis, Prentice-Hall, Englewood Cliffs, N. J., 1962. MR 24 \#A2844.

7. H. L. Royden, The boundary values of analytic and harmonic functions, Math. Z. 78 (1962), 1-24. MR 25 \#2190.

8. W. Rudin, The closed ideals in an algebra of analytic functions, Canad. J. Math. 9 (1957), 426-434. MR 19, 641.

9. E. L. Stout, On some algebras of analytic functions on finite open Riemann surfaces, Math. Z. 92 (1966), 366-379. MR 34 \#358.

10. J. Wermer, Analytic disks in maximal ideal spaces, Amer. J. Math. 86 (1964), 161-170. MR 28 \#5355.

11. M. Voichick, Ideals and invariant subspaces of analytic functions, Trans. Amer. Math. Soc. 111 (1964), 493-512. MR 28 \#4129.

WESLEYAN UNIVERSITY,

Middletown, Connecticut 06457 